\title{
Los retos jurídicos de la inteligencia artificial en el derecho en Colombia
}

\section{The legal challenges of artificial intelligence in law in colombia}

\author{
DOI: https://doi.org/10.17981/juridcuc.17.1.2021.17
}

Fecha de Recepción: 2020/08/04 Fecha de Aceptación: 2021/01/28

Tatiana Dulima Zabala Leal

Institución Universitaria Politécnico Grancolombiano. Bogotá, D.C. (Colombia). tzabala@poligran.edu.co

Paola Andrea Zuluaga Ortiz

Institución Universitaria Politécnico Grancolombiano. Bogotá, D.C. (Colombia). pazuluag@poligran.edu.co

Para citar este artículo:

Zabala, T. y Zuluaga, P. (2021). Los retos jurídicos de la inteligencia artificial en el derecho en Colombia. Jurídicas CUC, 17(1), 475-498. DOI: http://dx.doi.org/10.17981/juridcuc.17.1.2021.17

\section{Resumen}

En la actualidad la Inteligencia Artificial (IA) es un elemento que hace parte activa dentro de los sistemas jurídicos en diversos países del mundo, y en Colombia se han realizado algunas aproximaciones desde hace algunos años; lo anterior, debido a que el ser humano siempre ha pretendido crear herramientas que le faciliten la vida y que realice por él aquellas funciones operativas y procedimentales que le permitan dedicar su tiempo de trabajo a las actividades propias de la esencia de las ciencias o disciplinas en las que se desempeñan, y es precisamente allí, donde la tecnología ha jugado un papel trascendental en la creación de tipos de inteligencia que satisfaga esa necesidad procurando además generar un servicio a terceros. En razón a esto, el objetivo central de esta investigación fue describir el panorama legal de la IA, respecto de los actos jurídicos propios de los asuntos civiles, laborales, comerciales y administrativos en los que es parte y puede generar daños, en el contexto colombiano, para lo cual se utilizó una investigación documental, y como estrategia el análisis de contenido, generando como principal hallazgo la necesidad de generar un marco normativo alrededor de la responsabilidad de los actos $\mathrm{u}$ omisiones de los productos de la IA, la condición jurídica y el control de los mismos, teniendo como base fundamental los principios bioéticos.

Palabras Claves: Inteligencia artificial; robots inteligentes; autonomía; responsabilidad civil; principios bioéticos

\section{Abstract}

At present, Artificial Intelligence (AI) is an element that is an active part within the legal systems in various countries of the world, and in Colombia some approaches have been made for some years; The above, because the human being has always tried to create tools that make life easier for him and that perform for him those operational and procedural functions that allow him to dedicate his working time to the activities of the essence of the sciences or disciplines in those that are carried out, and it is precisely there, where technology has played a transcendental role in the creation of types of intelligence that satisfy this need while also trying to generate a service for third parties. Due to this, the central objective of this research was to describe the legal panorama of AI, with respect to the legal acts of civil, labor, commercial and administrative matters in which it is a party and can generate damages, in the Colombian context, for which a documentary research was used, and content analysis as a strategy, generating as the main finding the need to generate a regulatory framework around the responsibility of the acts or omissions of AI products, the legal status and the control of them, based on bioethical principles.

Keywords: Artificial intelligence; smart robots; autonomy; civil responsibility; bioethical principles 


\section{INTRODUCCIÓN}

En la actualidad la Inteligencia artificial es un elemento que hace parte activa dentro de los sistemas jurídicos en diversos países del mundo, y en Colombia ha realizado sus primeros ejercicios desde hace algunos años; lo anterior, debido a que el ser humano siempre ha pretendido crear herramientas que le faciliten la vida y que realice por él aquellas funciones operativas y procedimentales que le permitan dedicar su tiempo de trabajo a las actividades propias de la esencia de las ciencias o disciplinas en las que se desempeñan, y es precisamente allí, donde la tecnología ha jugado un papel trascendental en la creación de un tipo de inteligencia que satisfaga esa necesidad procurando además generar un servicio a terceros.

La Real Academia de la Lengua citada por Santos (2017), define la inteligencia como: "1. f. Capacidad de entender o comprender. 2. f. Capacidad de resolver problemas. 3. f. Conocimiento, comprensión, acto de entender. 4. f. Habilidad, destreza y experiencia" (p. 31). Por tanto, la inteligencia es un proceso cognoscitivo de razonamiento propio del ser humano, de esta actividad proviene la voluntad para la realización de actos jurídicos, el ejercicio de la capacidad y la causa exenta de vicios, de lo que derivan preguntas como: ¿Las obras de la IA son o no actos jurídicos que produce efectos en derecho?, ¿la IA goza de capacidad de ejercicio?, ¿la IA goza de autonomía de la voluntad?, ¿se genera responsabilidad civil contractual y extracontractual producto de las actividades realizadas en cabeza de la IA?, ¿de quién es la titularidad de los derechos de autor producto de las obras de la IA?, ¿quién responde civil o penalmente por los perjuicios a terceros producto de los actos de la IA?, entre otras.

En Colombia la implementación de la tecnología inteligencia artificial para la segmentación de las operaciones de los clientes en el sector comercial real y financiero se ha venido realizando desde hace no más de diez años y ha dejado como beneficio útiles sistemas de mitigación de riesgos para la prevención de actos de mala fe y pérdida económica. 
Además, uno de los grandes desafíos a los que se enfrenta la actual sociedad tecnológica o algorítmica está relacionado con el uso de la $I A$ y el alcance jurídico de sus actos, ya que estos modelos de inteligencia se utilizan para la puesta en funcionamiento de los bots, robots o androides, los cuales interactuaran con los humanos con cierto nivel de autonomía, siendo esta última, la capacidad de evolucionar a unidades que requieran cada vez una menor subordinación de sus fabricantes, propietarios o usuarios. Por lo tanto, se hace indispensable la promulgación de ordenamientos jurídicos que prevengan y mitiguen conflictos legales y sociales con la IA, delimitando su personalidad jurídica, definiendo la titularidad de los atributos de su personalidad, entre ellos su propiedad industrial y el régimen de responsabilidades a aplicarle.

Santos (2017) afirma que la inteligencia artificial y los productos derivados de la misma, son cada vez más complejos, ya que existen investigaciones que estudian la manera en que un robot, pueda concluir y prever las respuestas de los seres humanos y su habilidad para adecuarse o tomar decisiones por fuera de los parámetros establecidos ya sea por las leyes decretadas por la probabilidad, la estadística o algún patrón, lo cual implica una etapa de revolución, que conlleva consecuencias. Lo anterior, es corroborado por el Departamento Interior de Estados Unidos (DOI), quienes afirman que los robots inteligentes serán de uso común entre 2022 y 2027; y en el caso de Japón en el 2030, país que, en 2004, estableció unas zonas llamadas "Tokku", en las que se prueban robots en entornos reales (Kurita, 2020, p. 16). De igual manera, se considera que el mercado global de la robótica alcanzará los "44.48 millones de dólares para el año 2020” (Automática e Instrumentación, 2015, párr. 1).

Se puede decir, que a nivel tecnológico la humanidad ha pasado por diversas etapas; en la inicial, se abrió paso la internet y la conexión del ser humano a esta red, hasta llegar al lugar actual en el que las cosas se conectan automáticamente a la red en pro de optimizar la calidad de vida de las personas. Se espera que en la etapa futura las cosas, entendidas como robots, interactúen con su entorno de forma autónoma e independiente al control ejercido por los individuos, lo 
que implica la interacción de estos con el entorno de forma natural, incluyendo la probabilidad de que los seres humanos se ajusten con robots para perfeccionarse a lo que se le conoce como ciborg.

Teniendo en cuenta la relevancia de la robótica como producto del desarrollo de la IA, Santos (2017), estableció una serie de problemáticas que no han sido tenidas en cuenta en el desarrollo de la normatividad existente, como son:

- La multifuncionalidad de los robots, ya que su uso no se verá limitado a un solo campo de acción, sino que podrán ser utilizados en cualquier ámbito, incluso actualmente ya se encuentran en el mercado robots como ASIMO, que es un humanoide creado por Honda, con tecnología que le otorga un comportamiento autónomo; BUDDY, que funciona como compañía de humanos, desarrollado por Blue Frog Robotics, o ATLAS creado por Boston Dynamics, que ya va en su segunda generación y tiene como función principal el apoyo a los servicios de emergencia en operaciones de búsqueda y rescate; lo anterior, da muestra de que actualmente se está en la era robótica y está producirá paulatinamente nuevas necesidades humanas conforme a las características esenciales de la IA y al empleo que cada individuo le dé (Santos, 2017).

- Los sucesos originados en el hecho de, que si bien los robots realizan tareas asignadas a los seres humanos y se interrelacionen con ellos, estos no se desempeñan igual, pues no poseen intuición, ni conciencia, lo cual según Santos (2017) será generador de conflictos a nivel cultural, económico, social y legal; sobre todo en aquellos eventos en los que se requiera discernimiento subjetivo basado en el conocimiento general para la realización de actos jurídicos que impliquen responsabilidad legal.

- Es posible que se manifiesten vínculos emocionales con los robots por parte de los seres humanos con quienes interactúen, lo cual ha conllevado a afirmaciones como la hecha por Jiménez (2016), aludiendo un documento de la consultora Gartner donde se dice que "en el 2020 tendremos más pláticas con robots que con nuestras parejas", lo que según Santos (2017, p. 28) será el origen de riesgos psicosociales a causa de la incapacidad de diferenciar la realidad de la ficción. 
En razón a este tipo de riesgos, en algunas partes del mundo se ha considerado la posibilidad de generar una regulación específica para los casos de la biotecnología, la IA, la robótica y los aparatos pilotados a distancia; como es el caso de la Unión Europea (UE), la cual en 2015, genero un informe que recopila algunas recomendaciones dirigidas a la Comisión sobre Normas de Derecho Civil sobre Robótica (UE, 2015), con el objetivo "de asegurar que los robots estén o sigan estando al servicio de los seres humanos" (Santos, 2017, p. 28); entre estas se encuentran: la creación de una Agencia Europea de Robótica e IA; la elaboración de un Código de Conducta Ética Voluntaria; la definición de criterios de responsabilidad por los perjuicios producidos por los robots; la creación de un estatuto de persona electrónica; el contar con modelos de identificación del impacto social debido al aumento de los niveles de desempleo; la generación de principios de seguridad y privacidad para el desarrollo de la robótica y la IA; y la creación de un registro de robots inteligentes (Santos, 2017).

Siendo así, se plantea como pregunta eje de la investigación: ¿Cuáles son los principales cuestionamientos jurídicos, relacionados con el daño antijurídico que pueda causar la IA y/o sus respectivos productos?, del cual se deriva el objetivo general: Describir el panorama legal de la IA, en cuanto a los actos jurídicos, que tienen que ver con asuntos civiles, laborales, comerciales y administrativos en los que es parte y puede generar daños, en el contexto colombiano.

$\mathrm{El}$ anterior objetivo general se sustenta con los siguientes objetivos específicos: 1) Determinar que es la IA y que productos la comprenden; 2) Identificar la posible responsabilidad de los actos u omisiones de la IA y 3) Describir los conflictos a nivel ético que puede producir la convivencia del ser humano con los productos de la IA.

De esta manera, la presente investigación nace del requerimiento de ahondar en la identificación de los posibles efectos jurídicos de la interacción entre robots y humanos, y del aumento progresivo de la autonomía de estos en la relación directamente proporcional con sus fabricantes, propietarios y usuarios, la cual implica una disminución progresiva de la dependencia; lo que permitirá evidenciar que se requiere de forma imperiosa la determinación de la condición jurídica 
de la IA, su régimen de derechos y deberes, su código de ética y los aspectos a tener en cuenta para la solución de los problemas derivados de sus actos; además, de establecer los posibles riesgos de seguridad que se dan al usarla; siendo la normativización de todo lo anterior la clave para que esta coexistencia sea lo más pacifica posible.

La consciencia, de la necesidad de regulación jurídica de los productos de la IA, ha venido en un proceso de construcción ascendente. Por su parte, UE, Japón y Estados Unidos, ya han dado algunas directrices como la creación del Grupo de Evaluación de las Opciones Científicas y Tecnológicas STOA, en 2015, el cual realizo un estudio prospectivo titulado "Aspectos éticos de los Sistemas ciber físicos" (UE, 2016); sin embargo, en el caso colombiano se observa una falencia y un desconocimiento acerca de la necesidad de la regulación normativa para las relaciones de los robots y los humanos en un futuro cercano.

\section{Desarrollo}

\section{Fundamentación teórica}

\section{- Inteligencia artificial}

La IA, se está volviendo cada vez más poderosa ya que existen una gran variedad de productos y servicios digitales tanto en el contexto público como en el privado, los cuales han venido siendo patentados legalmente, y en combinación con los datos, la ciencia, la tecnología de sensores, el internet, la mecánica y la ingeniería eléctrica, entre otros, están generando sistemas robóticos de alta tecnología de utilidad en el sector productivo, la salud, el comercio, el hogar la seguridad y la protección (European Group on Ethics in Science and New Technologies-EGE, 2018).

En razón a lo anterior, autores como Granell (2016), afirman que estamos en la cuarta revolución industrial, ya que la internet, la IA y la robótica están modificando las rutinas diarias del comportamiento humano, puesto que la investigación en estas áreas avanza rápidamente. Lo que implica que la sociedad se está viendo direccionada hacia la convivencia con los robots y la IA. 
Una prueba de esto, es que hoy por hoy son más las empresas que le apuestan al uso de la IA y de la Big Data como herramientas para obtener una mayor precisión y rapidez en sus resultados. Gutiérrez (2017) afirma que son los sectores que la utilizan los que más tarde se verán afectados por la misma; sin embargo, el elemento de la cercanía desde la psicología humana que tanto es valorado y estudiado, por ahora, sostiene que "no es previsible que puedan hacerlo las máquinas" (García citado por Salazar, 2018, p. 305). "Respecto a las regiones hay países más propensos a la implantación exitosa de las nuevas tecnologías" (Hawksworth, Berriman and Cameron, 2018, p. 7).

Todo lo anterior, corrobora lo expuesto por Penrose (1996), quien afirmaba que la IA viene en un proceso de constante construcción desde los contextos computacionales de Tuning hasta la robótica y los sistemas expertos. Convirtiéndose en un contexto donde convergen lo técnico y lo científico, ya que "busca comprender, ajustar y responder inteligencia y métodos cognitivos, implicando variables matemáticas, lógicas, mecánicas y elementos y progresos biológicos" (Frankish \& Ramsey, 2015. p. 3).

De acuerdo con Cairo (2011), el escenario en el que se desarrolla la IA es el "entendimiento desde la perspectiva científica de los componentes que cimentan el pensamiento y la conducta humana inteligente y su inscripción en las máquinas" (p. 3). Estos dispositivos que menciona Cairo (2011), manejan conceptos como bio-vida, información, conocimiento, y nano o simplicidad, los cuales son comparables con la biotecnología, la información tecnológica, la ciencia cognitiva y la nanotecnología; siendo el objetivo de estos "reconocer el grado de correlación y amplitud de las máquinas en un argumento particularmente alterno al servicio del desarrollo humano" (Henk, 2007, p. 82).

Por otra parte, para hablar de IA, se debe iniciar desde la inteligencia como la competencia de tratar información para la solución de problemas en pro de alcanzar objetivos (Gardner, 2010). Esta noción se encuentra vinculada al procesamiento de la información desde el enfoque cognitivo, donde el flujo de esta y del contexto, 
se debe codificar, organizar, seleccionar, almacenar y recuperar por medio de los sistemas sensoriales, perceptivos, etc. (Corvalán, 2018).

De esta manera, la inteligencia se relaciona con algunas habilidades cognitivas autónomas que son clasificados como "inteligencias múltiples", las cuales son: social, lingüística, lógico-matemática, interpersonal, emocional y fluida entre otras (Corvalán, 2018, p. 230). Lo anterior, implica que el cerebro interviene en la capacidad de proceso de la información proveniente del entorno y del mismo cuerpo humano (Gerard y Gerald, 2015). Que es utilizada en la evaluación y elección de alternativas, entrando al juego de la toma de decisiones y de la evaluación, entendida esta última en la selección, recorte y organización de la información aprovechable (Corvalán, 2018).

Con base en la inteligencia humana se han venido desarrollando diversos procesos innovadores a nivel tecnológico como los que son utilizados en el procesamiento de la información en la solución de problemas y en la toma de decisiones a través de máquinas que son operadas por algoritmos inteligentes, los cuales son el sustento de la IA y que son empleados para identificar tendencias económicas, predicción de delitos, diagnóstico de enfermedades, predicción de comportamientos digitales entre otros (Corvalán, 2018). Y son definidos por Domingos (2015), como un "grupo exacto de normas o una sucesión sistemática de pasos que puede usarse para realizar cálculos, solucionar problemas y tomar decisiones. El algoritmo es la fórmula que se usa para hacer un cálculo" (p. 1).

Todo lo anteriormente expuesto, implica que la aplicación de la IA, tiene como propósito permitir que los sistemas computacionales posean: autodependencia, reconfiguración adaptativa, negociación inteligente, comportamiento de cooperación y supervivencia con intervención humana reducida entre otras características (Barrat, 2015). Situación que implica el uso de técnicas fundamentadas en la identificación de modelos para la resolución de problemas (Serrano, 2016), así como también la maximización de objetivos y al optimización del procesamiento de la información (Corvalán, 2018). 


\section{- La normativa europea de la IA}

La Unión Europea (UE), a partir de la Estrategia Global para la Política Exterior y de Seguridad (UE, 2016) establece la necesidad de generar reglas mundiales en contextos como la biotecnología, la IA, la robótica y los aparatos pilotados a distancia, con el solo objetivo de eludir conflictos de seguridad y fructificar los beneficios económicos obtenidos de estas actividades, conexo a esto la UE ha diseñado foros multilaterales dispuestos a la elaboración de normas y asociaciones para la regulación multilateral (Instituto Complutense de Estudios Internacionales-ICEI, 2018).

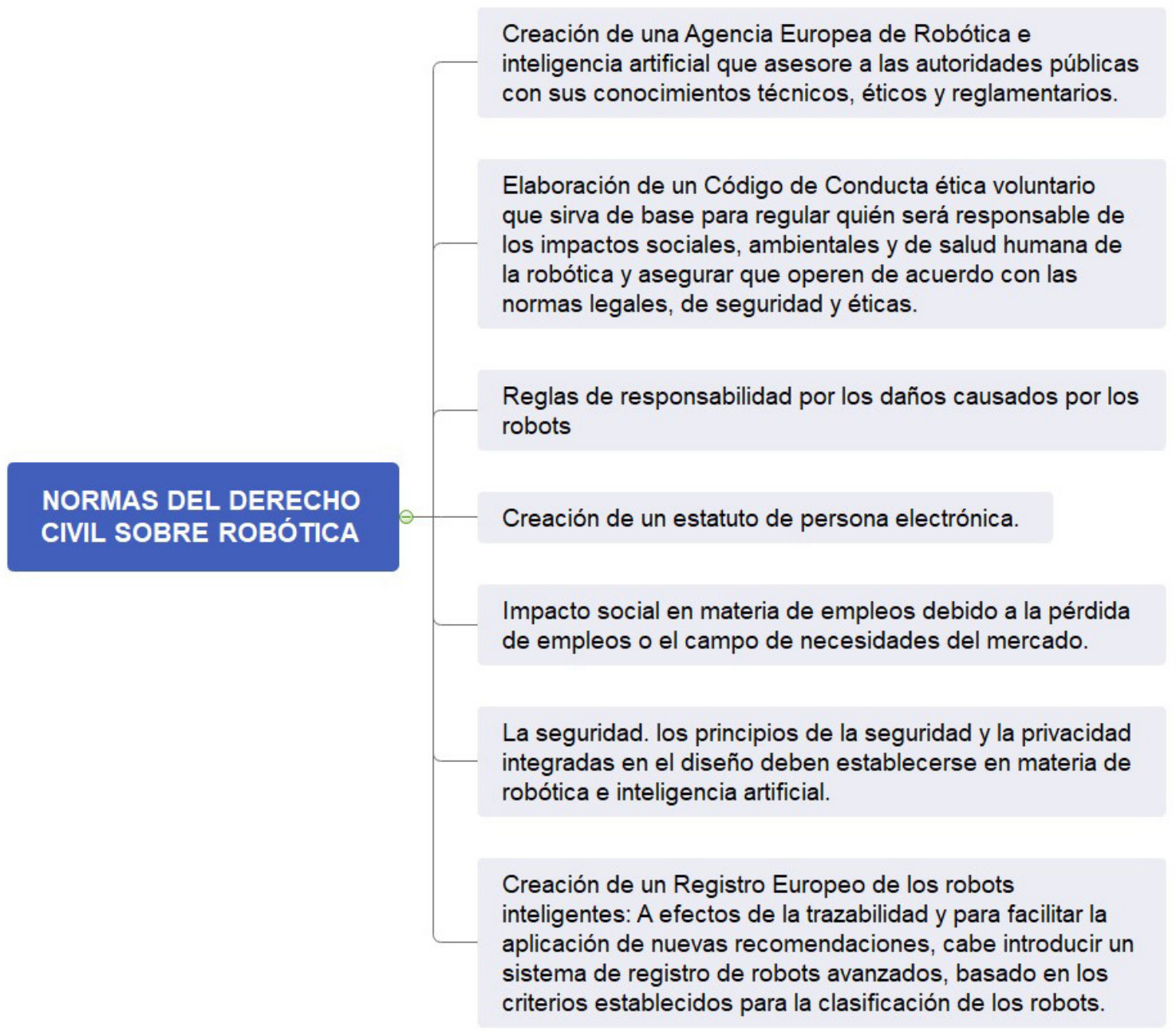

Figura 1. Normas sobre del derecho civil sobre robótica.

Fuente: Elaboración propia con datos de Parlamento Europeo (EPRS,2017). 
Con miras al anterior objetivo, el punto de partida se dio en el informe del 31 de mayo de 2016, en el que se compilan las sugerencias hechas a la Comisión Sobre Normas de Derecho Civil sobre Robótica para certificar que los robots siempre estén al servicio de los seres humanos; informe que fue aprobado el 16 de febrero de 2017 por el Parlamento Europeo (EPRS, 2017), en el que se dan las líneas primordiales de trabajo a ser tenidas en cuenta para la legislación de la robótica, las cuales se muestran en la Figura 1.

La gran preocupación no solo en Europa, sino a nivel global, se encuentra en los conflictos éticos que se puedan dar en diferentes momentos con la utilización generalizada de la IA, razón por la que en 2015 se creó el Grupo de Evaluación de las Opciones Científicas y Tecnológicas-STOA, cuya función es apoyar al órgano legislador europeo, este grupo realizo un estudio llamado "Aspectos éticos de los sistemas ciber físicos", en el cual se exponen los diversos conflictos a nivel ético que están vinculados con la tecnología proyectados al 2050 y además clasifica la tecnología de acuerdo a las áreas de aplicación, planteando dilemas morales que convendrían ser abordados por el legislador y propone como temas jurídicos a desarrollar el compromiso, la seguridad, la concepción jurídica de los robots, la propiedad de los datos y la privacidad (EPRS, 2016).

\section{- Retos a nivel normativo que presenta la IA}

El primero de los retos planteados a nivel normativo por el uso de la IA, es la conceptualización de los Robots Inteligentes, ya que actualmente los robots operan a través de instrucciones humanas y realizan tareas automáticas y complicadas. Sin embargo, los robots inteligentes podrán coordinas diversas acciones e interactuar con el contexto en el cual se desenvuelven, para afrontar esta situación la sociedad debe asignar una definición concreta para el termino robot inteligente, lo cual se dificulta ya que todavía se discute el concepto de persona y en qué momento determinado el embrión adquiere esa posición; y según Santos (2017), existen diversas 
aproximaciones al concepto de robot, más ninguna está registrada o ha sido tomada de manera consensuada a nivel internacional ni europeo.

El Parlamento Europeo afirma que, para definir al robot inteligente, se deben tener en cuenta las siguientes características: a) La disposición de tener autonomía ya sea por el componente de sensores o por el cambio de datos con su entorno o interconectividad, acompañado con su respectivo estudio; b) capacidad de formarse por medio de la experiencia y la interacción; c) el soporte físico del robot; y d) la idoneidad para adaptar su comportamiento y acciones al entorno (EPRS, 2015).

El reto normativo consiste en llegar a una definición del robot inteligente, teniendo en cuenta la polémica acerca de si pueden llegar a ser conscientes de su propia existencia y poder experimentar sentimientos análogos a los humanos.

Un segundo reto, es la ciberseguridad la cual se ha venido construyendo como un derecho de los ciudadanos y un deber del Estado garantizarla a través de la promoción de medios de seguridad y aseguramiento de la integridad de las infraestructuras y la información, además, las diferentes problemáticas vinculadas a la IA, están relacionadas con los axiomas fundamentales de la ciberseguridad que son la confidencialidad, la integridad y la disponibilidad, razón fundamental para que el desarrollo tecnológico sea acompañado de medidas de seguridad adecuadas para evitar disturbios (Santos, 2017).

El tercer desafío se da desde la responsabilidad por las decisiones, actos $\mathrm{u}$ omisiones de los robots, que se da desde el supuesto de la utilización masiva de los robots inteligentes, acrecienta la probabilidad de ocurrencia de daños o perjuicios originados en las omisiones de estos, y se agudiza por la falta de límites, condiciones y responsabilidades generando los siguientes interrogantes: ¿Una maquina puede visualizarse como parcial o totalmente responsable de su conducta? Y, ¿se pueden encuadrar a los robots inteligentes en una de las categorías jurídicas existentes como son las personas naturales o físicas, las personas jurídicas, animales u objetos? 
Lo anterior, se encuadra en la responsabilidad civil, que se origina en el principio del derecho que establece la obligación de no perjudicar a otro de manera injusta neminem laedere y que se aplica a toda persona que desee vivir en sociedad. El alcance de esta responsabilidad incluye el cumplimiento de obligaciones concretas, ya sea que se hayan adquirido de manera voluntaria, o que sean impuestas por norma legal; quien incumple este deber general, incurre en la obligación de indemnizar los daños causados (Tamayo, 2006).

El objetivo del concepto anterior, es el restablecimiento del equilibrio económico de la victima antes de habérsele ocasionado el perjuicio. Según la Corte Constitucional colombiana, para que resulte comprometida la responsabilidad de una persona natural o jurídica, se necesitan de tres elementos que son : culpa, daño y relación de causalidad; las que definen la carga probatoria del demandante, quien posee el deber de demostrar el detrimento patrimonial o moral que la conducta culpable del demandado origino, razón por la que la responsabilidad se enmarca en una relación jurídica entre el autor del daño y quien lo padeció (Sentencia C-1008, 2010).

Bajo este marco normativo, en la actualidad, los robots no pueden ser considerados responsables por los actos $u$ omisiones que sean originadores de daños a terceros, lo cual se fundamenta en el hecho de que a pesar de poseer autonomía y el auto aprendizaje, hay múltiples actores que participan con el robot, como son el programador, el fabricante, el operador, el comprador, el propietario, el usuario entre otros, razón por la que no es posible determinar exactamente que parte debe hacerse cargo de la indemnización o de la reparación del daño causado, situación que lleva a plantear el siguiente interrogante: ¿Es posible responsabilizar al robot por los actos $\mathrm{u}$ omisiones que no puedan ser atribuidos a los seres humanos o a estos mismos por los actos $u$ omisiones de los robots al causar daños que podrían haberse evadido?

Por tanto, desde la perspectiva técnica, puede ser inalcanzable la justificación de los motivos de una decisión concreta de IA, 
encontrando que en el caso de los daños, las partes se encuentran con un vacio probatorio y se les imposibilita determinar la responsabilidad al carecer de una normativa particular y especifica para estos casos (Čerka, Grigienè \& Sirbikytè, 2017).

El cuarto desafío, que se tuvo en cuenta en esta investigación es la naturaleza jurídica de los robots, ya que no está claro a que categoría jurídica corresponden, lo que genera las siguientes preguntas: ¿Los robots inteligentes se pueden vincular a las categorías de personas físicas o naturales, personas jurídicas, animales u objetos? $\mathrm{O}$, ¿debe crearse una categoría que este limitado por una característica jurídica especificas?

Al respecto, Puyol (2018) afirma que se está adportas de una nueva figura llamada "persona electrónica" que se encuentra en un punto intermedio entre las físicas y las cosas, proceso que debería ser similar a el momento en que se les concedieron derechos a las personas jurídicas.

\section{MetodoloGía}

Esta investigación, se ubicará en el paradigma interpretativo (Histórico-Hermenéutico), ya que en este "el investigador trata de descubrir el significado de las acciones humanas y de la vida social, dirige su labor a entrar en el mundo personal de los individuos, en las motivaciones que lo orientan, en sus creencias" (González, 2003). Lo que es coherente con el objetivo principal que es "Describir el panorama legal de la IA, en cuanto a los actos jurídicos, que tienen que ver con asuntos civiles, laborales, comerciales y administrativos en los que es parte y puede generar daños, en el contexto colombiano".

El proyecto acá planteado se encuentra ubicado en los estudios culturales e interpretativos, puesto que se abordan realidades subjetivas e intersubjetivas, como objetos legítimos de conocimientos científicos como lo es la inteligencia artificial, apuntando a la comprensión de la realidad relacionada con los actos jurídicos dados en relación con el objeto de conocimiento, como resultado de un proceso histórico de construcción a partir de las lógicas de los auto- 
res, reconociendo la subjetividad como condición de conocimiento, la validez estratégica con un fin, la confiabilidad de las fuentes, la sistematicidad o construcción teórica y la comunicabilidad mediante múltiples lenguajes, donde se da cabida a diferentes apropiaciones conceptuales y metodológicas que se ofrece al investigador como cajas de herramientas que no están limitadas por criterios académicos, sino por la realidad social.

Por tanto, desarrollar los objetivos planteados para el desarrollo de esta investigación, implica analizar la literatura alrededor de los temas centrales de la misma. Para lograrlo se tuvo como estrategia metodológica la investigación documental descrita por Galeano y Vélez (2002), como un método con características propias en lo que respecta a la obtención de información, el análisis e interpretación, ya que combina fuentes diversas al utilizar fuentes secundarias recolectadas a través de archivos oficiales o privados los cuales son su materia prima.

La estrategia estuvo apoyada en una revisión sistemática de literatura como técnica de búsqueda, cuyo objetivo será el rastreo, la ubicación, inventariar, seleccionar y consultar las fuentes y los documentos que se van a utilizar como materia prima en la investigación.

Finalmente, como técnica de análisis, se utilizó el análisis de contenido, ya que permitió analizar el material bibliográfico para este caso desde la perspectiva cualitativa, el procedimiento de este análisis fue direccionado por Martínez (2004), quien propone cuatro momentos para la teorización: el primero la categorización, la cual inicia con la transcripción de la información protocolar, pasando por el establecimiento de unidades temáticas, para luego codificar; el segundo momento es al estructuración, donde se presenta una síntesis descriptiva de los hallazgos; el tercer momento es la contrastación, en el que se utilizara la triangulación para comparar los resultados con los estudios paralelos o similares que se presentaron en el marco teórico referencial; y finalmente la teorización, en donde se tratara de integrar en un todo coherente y lógico los resultados de la investigación. 


\section{Resultados}

\section{Inteligencia Artificial}

A través de la revisión bibliográfica, se determinó como la definición que más poseería utilidad a nivel jurídico es la de Malpica (2016), quien afirma que la IA, es una "rama de las ciencias de la computación que estudia el software y hardware necesarios para simular el comportamiento y comprensión humanos" (p. 1). Cuyo objetivo es la imitación de la inteligencia humana en una máquina, utilizados generalmente para la construcción de robots que tengan conciencia y sentimientos parecidos a los de los seres humanos; siendo su mayor obstáculo precisamente la generación de la primera ya que es una cualidad explícitamente humana.

\section{Productos de la $I A$}

Como productos de la IA, se encuentran las diferentes aplicaciones de estas como son: la robótica entendida como la rama de la tecnología que estudia el diseño y la construcción de máquinas conocidas como robots que son capaces de ejecutar tareas que normalmente son realizadas por el ser humano o necesitan del uso de la inteligencia (Vector ITC, 2018). Siguiendo se encontró la simulación experimental, proceso por el que, a través de la combinación matemática, se organizan grupos de sujetos para probar el efecto de una variable independiente en una dependiente, lo que en al IA significa realizar una simulación experimental a través del ordenador para reproducir el comportamiento de un proceso cognitivo real (Vector ITC, 2018).

Además, están los RPA (Robotic Process Automation), que son sistemas de automatización de procesos, o tecnología de rápido crecimiento que permite a las organizaciones implementar sistemas de software inteligentes que tienen la propiedad de imitar las acciones de los humanos (Vector ITC, 2018). También se encuentran los asistentes virtuales, cuya función principal es ayudar a los usuarios a aclarar dudas y realizar acciones específicas, como son la reserva de billetes, la compra de productos y servicios o la consulta de información (Vector ITC, 2018). 
Por último, están las redes neuronales y los sistemas de aprendizaje, las primeras imitan las funciones de las de los organismos vivos, generando un conjunto de neuronas conectadas entre sí, que trabajan en conjunto y son parte de los procesos fundamentados en Machine Learning. Y los segundos, son utilizados generalmente en el campo del E-Learning, permitiendo crear sistemas educativos y herramientas de aprendizaje personalizadas, enfocadas a los alumnos, con la capacidad de adaptarse al ritmo de estudio de estos y los ayudan a desarrollar el proceso de aprendizaje adecuadamente (Vector ITC, 2018).

\section{Responsabilidad de los actos u omisiones de la IA}

A través de la revisión documental, se encontró la existencia de deficiencias en el marco normativo en lo que respecta a la responsabilidad contractual, ya que la existencia de las aplicaciones de la IA que realizan actos similares a los humanos, que además tienen la opción para tomar decisiones, hace que la normativa tradicional generalmente surgida del Código Civil Colombiano sea inaplicable, ya que el campo de acción de la legislación se limita al proceso de fabricación y comercialización, desde la perspectiva de las características de seguridad y ética, ya que estos adquieren una responsabilidad jurídica con respecto al factor calidad de la tecnología producida.

Además, se encuentra en la investigación un factor relevante, que es la perspectiva de que, entre los productos de la IA, que más tiene y tendría a futuro interacción con el ser humano son los robots, que, a pesar de ser seres inertes, en el momento en que se conjugan con la IA, pasan a ser sujetos inmersos en comunidades y seres capaces de realizar actos, que en un momento dado pueden ocasionar daños, que podrían haber sido advertidos y prevenidos por parte de sus creadores.

Esta limitación, es contraproducente, puesto que no es correcto limitar el alcance de los daños o perjuicios que puedan ser objeto de una compensación, o mucho menos de la naturaleza de esta, solo porque estos son causados por robots y no por un ser humano. 
En razón a que hay múltiples actores que participan con el robot, como son el programador, el fabricante, el operador, el comprador, el propietario, el usuario entre otros, se debería hablar de la responsabilidad en la gestión de riesgo, pero no centrada en la persona que fue negligente en su actuación, sino en aquella que tiene la función de minimizar los riesgos y el impacto negativo, lo cual implica en un primer momento identificar claramente la responsabilidad de cada uno de los actores; esta debería ser proporcional al nivel de las instrucciones dadas a los robots y al nivel de autonomía dado, por tanto, se establecería una relación directamente proporcional a mayor autonomía, mayor es la responsabilidad de cada actor formador y de esta manera de acuerdo a la normativa actual la responsabilidad debe ser asumida por un humano, ya que los robots todavía no tienen una clasificación exacta y existe una constante polémica al respecto.

Santos (2017) propone ante esta situación, en vista de lo complicado que seria el proceso de asignación de responsabilidad por los daños y perjuicios causados por los robots; el establecimiento de un régimen de seguro obligatorio sumado a un fondo común cuyo objetivo sea cubrir los riesgos, lo cual es coherente con algunas de las propuestas de la Comisión Europea.

\section{Conflictos éticos en la convivencia del ser humano y la IA}

Las ventajas de la IA, son muchas y aportan soluciones tecnológicas a muchos campos, no obstante, a través de la revisión documental realizada se encuentran diversos factores de riesgo originados en el cuestionamiento ético de los programadores de IA, del desarrollo de ella misma, y de la privacidad de los datos de los usuarios, lo que es confirmado por Salazar (2018), quien afirma que los algoritmos poseen la función de aprender de lo que hacen, es decir, que toman información del desarrollo de su trabajo y de su contexto, generando la posibilidad de que en un momento dado pueden desvincularse de su programación inicial (heurística).

En razón a la situación planteada anteriormente y a otras que se pueden suscitar desde la bioética surge el transhumanismo, que es ante todo un escenario científico que tiene por objetivo "cuestionar 
la posibilidad y conveniencia en la transformación de la condición humana, creada y desarrollada mediante tecnologías que disminuyan el envejecimiento de la persona y aumenten su capacidad intelectual, física, y psicológica" (Russell \& Norving, 2003, p. 58).

Este escenario, se da desde diversas perspectivas como el hecho de que a través de la IA el ser humano se enfrenta a realidades contradictorias, como la potenciación de la libertad individual al usar la tecnología, sin embargo, a la vez se sufre la fragmentación social y el aislamiento, sumados a los problemas ecológicos y políticos a nivel del planeta, lo que plantea una incapacidad de afrontarlos desde criterios y valores universales ya que hay un escepticismo moral en la confrontación ética de la utilización de la IA.

De tal manera, que la parte positiva de la utilización de la IA, es la potencialización de la capacidad de creación, así como la generación de posibilidades humanas, generando alternativas de desarrollo y bienestar, sin embargo, desde la parte negativa se generan poderes como el control, el direccionamiento a la uniformidad y al destrucción de los limites naturales; que generan consecuencias inciertas, las cuales han hecho que se construya el concepto de "ética de la IA", expuesto por Bostrom y Yudkowsky (2014), que proponen crear maquinas pensantes circunscritas a un marco ético, desde el punto inicial de su creación sin que se rompa el principio de la beneficencia de la bioética ni conflictos en el estatus moral de las aplicaciones emergidas de la IA.

Esta posición desde la bioética implica, un acompañamiento de estas teorías en la generación de normativas acerca del uso de la IA, ya que también acompañan la posición de muchos autores acerca de la distribución de responsabilidades de los productores y beneficiarios de la misma, por lo que Bostrom y Yudkowsky (2014) proponen una genuina y cualificada equidad en su aplicación — determinada por un panel de jueces humanos - escenario donde un algoritmo es aceptado en una aplicación transparente y rechaza negras intenciones. Razón por la cual, se recomendaría a la comunidad académica realizar estudios sobre la generación de propuestas normativas que tengan en cuenta los principios bioéticos aplicados a la IA. 


\section{Conclusiones}

A través del presente ejercicio de investigación, se confirma la posibilidad de la convivencia de los robots con los seres humanos en el contexto colombiano, no obstante, se identificaron como situaciones riesgosas a las cuales la normativa debe apoyar : desconfianza en el sistema de evaluación de los robots, la imposibilidad de prevenir la conducta de los mismos, la falta de estandarización en los procesos de evaluación, la falta de claridad en la condición jurídica de los robots y las limitantes que deberían establecerse para aquellos robots que poseen la capacidad de la autonomía.

Además, hay algunos factores y conceptos que se deben analizar paralelamente al diseño de una normativa correspondiente a la IA, como son la autonomía, el aprendizaje, la conciencia, la evaluación, el libre albedrio, el razonamiento, la capacidad de comprender, el proceso de toma de decisiones, la libertad, las emociones, la inteligencia tanto en los seres humanos pertenecientes a una comunidad, sino también en aquellos productos de la IA, que tienen la posibilidad de interactuar con ellos; puesto que en algún momento determinado una palabra no tendrá el mismo significado en las dos categorías.

Por otra parte, en lo que respecta al sector productivo del país, el cual es uno de los principales usuarios de la IA, se deben implementar normas, esquemas y procedimientos que atiendan los aspectos particulares en temas éticos, legales, de organización, técnicos, de control y operativos; puesto que cualquier falla conlleva la posibilidad de ocasionar víctimas, daños materiales, alteraciones en la producción, afectando de esa manera la información financiera de la empresa y en muchos casos de acuerdo a la naturaleza de la empresa, se puede poner en riesgo hasta la vida de los individuos.

Otro factor relevante que se observa, es el hecho de que la información personal, al producirse la vinculación de productos derivados de la IA, será mas vulnerable ya que se presentara una transmisión de la información, conduciendo a los legisladores a construir limites en lo que respecta al derecho a la privacidad, ya que es un proceso itinerante entre la privacidad de las personas y los productos de la IA. 
Razón por la cual, en el panorama colombiano se debe revisar la normativa acerca de la responsabilidad de sus actos $\mathrm{u}$ omisiones, y además su condición jurídica, el ser o no sujetos controlados y de ser así, cual o cuales serían las maneras de hacerlo, incluyendo los principios de la bioética como elementos necesarios para garantizar la seguridad de los usuarios y poder aprovechar las ventajas que su uso puede traer a la sociedad colombiana. En la estructuración de esta normativa se deben definir principios y axiomas fundamentales que resuelvan los problemas éticos, legales y de seguridad originados de la aplicación de la IA, involucrando a científicos, empresarios gobernantes y ciudadanos.

\section{REFERENCIAS}

Automática e Instrumentación. (sept. 15, 2015). El mercado de la robótica industrial. [Online]. Disponible en http://www.automaticaeinstrumentacion.com/es/notices/2015/09/el-mercado-de-la-robotica-industrial-43161.php\#.Xh-zhshKiUl

Barrat, J. (2015). Nuestra invención final. Madrid: Planeta Publishing.

Bostrom, N. \& Yudkowsky, E. (2014). The ethics of artificial intelligence. Cambrigde University Press: The Cambridge handbook of artificial intelligence. Disponible en https://intelligence.org/files/EthicsofAI.pdf

Cairó, O. (2011). El hombre artificial: el futuro de la tecnología. México, D.F.: Alfaomega.

Čerka, P., Grigienè, J. \& Sirbikytè, G. (2015). Liability for damages caused by artificial intelligence. Computer Law \& Security Review, 31(3), 376-389. https://doi.org/10.1016/j. clsr.2015.03.008

Corvalán, J. (2018). Inteligencia artificial: retos, desafíos y oportunidades - Prometea: la primera inteligencia artificial de Latinoamérica al servicio de la Justicia. Revista de Investigações Constitucionais, 5(1), 295-316. https://doi. org/10.5380/rinc.v5i1.55334 
Domingos, P. (2015). The máster algorithm: how the quest for the ultimate learning machine will remake our world. New York: Basic Books.

EC. EGE. (2018). Statement on Artificial Intelligence, Robotics and 'Autonomous' Systems, European Group on Ethics in Science and New Technologies. Brussels: CE. Disponible en https://op.europa.eu/en/publication-detail/-/publication/dfebe62e-4ce9-11e8-be1d-01aa75ed71a1

Frankish, K. \& Ramsey, W. (2015). The Cambridge handbook of artificial intelligence. Cambrigde: Cambrigde University Press. https://doi.org/10.1017/CBO9781139046855

Galeano, M. y Vélez, O. (eds.) (2002). Investigación cualitativa estado del arte. Estado del arte sobre fuentes documentales en investigación cualitativa. Medellín: Universidad de Antioquia.

Gardner, H. (2010). La inteligencia reformulada. Madrid: Paidós.

Gerard, M. y Gerald, G. (2015). El libro de la biología. Madrid: Ilus Books.

González, A. (2003). Los paradigmas de investigación en las ciencias sociales. ISLAS, 45(138), 125-135. Recuperado de http://biblioteca.esucomex.cl/RCA/Los\%20paradigmas\%20 de\%20investigaci\%C3\%B3n\%20en\%20las\%20ciencias\%20 sociales.pdf

Granell, F. (2016). Los retos de la cuarta revolución industrial. En, Real Academia de Ciencias Económicas y Financieras, Perspectivas económicas frente al cambio social, financiero y empresarial. Solemne acto académico conjunto con la Universidad de La Rioja y la Fundación San Millán de la Cogolla (pp: 57-74). Barcelona: RACEF. Recuperado de https://racef.es/archivos/publicaciones/web_racef_larioja_44.pdf

Gutiérrez, Á. (noviembre 22, 2017). Yo, Robot: puestos de trabajo que van a desaparecer. [Online]. Disponible en https:// ecommerce-news.es/robot-puestos-van-desaparecer/ 
Hawksworth, J., Berriman, R. and Cameron, E. (2018). Will Robots really steal our jobs?: An international analysis of the potential long term impact of automation. London: Price Water house. Recuperado de https://www.pwc.co.uk/economic-services/assets/international-impact-of-automation-feb-2018. pdf

Henk, A. (ed). (2007). Nanotechnologies, ethics and politics. Ethics series. Paris: Unesco publishing. Disponible en https://unesdoc.unesco.org/ark:/48223/pf0000150616

ICEI. (2018). La Estrategia Global y de Seguridad de la Unión Europea: narrativas securitarias, legitimidad e identidad de un actor en crisis. [Online]. Disponible en https://www.ucm.es/ data/cont/media/www/pag-28960/WP\%2001\%20final.pdf

Jiménez, A. (noviembre 8, 2016). 10 Tendencias de Transformación Digital: espacios para la gestión del cambio. FuncionC. Disponible en https:/funcionc.com/2016/11/08/10-tendencias-de-transformacion-digital-espacios-para-la-gestion-delcambio/

Kurita, M. (2020). Accidentes de Robots y responsabilidad civil. Revista de la Sociedad de Robótica de Japón. 38(1), 41-46. 2020. https://doi.org/10.7210/jrsj.38.41

Malpica, J. (2016). Inteligencia Artificial y Conciencia. Departamento de Matemáticas de la UAH, en el área de conocimiento Ingeniería Cartográfica, Geodesia y Fotogrametría. [Online]. Disponible en http://www3.uah.es/benito_fraile/ponencias/inteligencia-artificial.pdf

Martínez, M. (2004). Ciencia y arte en la metodología cualitativa. México, D.C.: Trillas.

Penrose, R. (1996). The emperor's new mind. México, D.F.: FCE. Editorial Oxford University Press.

Puyol, J. (diciembre 17, 2018). Robots: ¿Hacia un nuevo concepto de "personalidad jurídica»? confilegal.com. Disponible en https://confilegal.com/20181217-hacia-un-nuevo-conceptode-personalidad-juridica/ 
República de Colombia. Corte Constitucional. (9 de diciembre de 2010). Exp. D-8146. [Sentencia C-1008-10]. Magistrado Ponente: Luis Ernesto Vargas Silva. Disponibleen https://www. corteconstitucional.gov.co/RELATORIA/2010/C-1008-10. htm

Russell, S. \& Norvig, P. (2003). Artificial intelligence: A modern approach. (2 ed.). New Jersey: Prentice Hall.

Salazar, I. (2018). Los robots y la Inteligencia Artificial. Nuevos retos del periodismo. Doxa Comunicación, (27). 295-315. https://doi.org/10.31921/doxacom.n27a15

Santos, M. (2017). Regulación Legal de la Robótica y la Inteligencia Artificial: Retos de Futuro. Revista Jurídica de la Universidad de León, (4), 25-50. Disponible en http://revpubli. unileon.es/index.php/juridica/article/view/5285/4108

Serrano, A. (2016). Inteligencia artificial. Madrid: RC.

Tamayo, J. (2006). La responsabilidad civil en el código de comercio. Revista de la Facultad de Derecho y Ciencias Politicas, (72), 59-88. Disponible en https://revistas.upb.edu.co/ index.php/derecho/article/view/5045

UE. (2016). Shared vision, common action: A stronger Europe. A global strategy for the European Union's foreign and security policy. [Online]. Luxembourg: Office of the European Union. Disponible en https://op.europa.eu/en/ publication-detail/-/publication/3eaae2cf-9ac5-11e6-868c01aa75ed71a1

UE. EPRS. (2017). Normas de Derecho civil sobre robótica. [P8_TA (2017)0051]. Disponible en https://www.europarl.europa. eu/doceo/document/TA-8-2017-0051_ES.pdf?redirect

UE. EPRS. (2016). Scientific Foresight study Ethical Aspects of Cyber-Physical Systems. Scientific Foresight Unit. [STOA]. Brussels: Panel para el Futuro de la Ciencia y la Tecnología. Recuperado de https://www.europarl. europa.eu/RegData/etudes/STUD/2016/563501/EPRS_ STU\%282016\%29563501_EN.pdf 
UE. EPRS. (2015). Proyecto de Informe con recomendaciones destinadas a la Comisión sobre normas de Derecho civil sobre robótica. [2015/2103(INL)]. Brussels: Comisión de Asuntos Jurídicos. Disponible en https://www.europarl.europa.eu/ doceo/document/JURI-PR-582443 ES.pdf?redirect

Vector ITC. (2018). Inteligencia artificial: pasado, presente y futuro. [Online]. Disponible en https://www.vectoritcgroup.com/ wp-content/uploads/2018/06/IA-Pasado-presente-y-futuroVector-ITC.pdf

"El presente artículo está adscrito al proyecto de investigación denominado" Marco normativo de las industrias creativas, tecnología, contratos y arbitraje en Colombia" de la Institución Universitaria Politécnico Grancolombiano.

Tatiana Dulima Zabala Leal es Magíster en Derecho comercial y contratos internacionales, Candidata a título de Doctorado en Derecho empresarial, Abogada, Docente Investigadora, Grupo de Investigación Derecho Sociedad e Industrias Creativas, Escuela de Derecho y Gobierno, Línea de Investigación Derecho de la Información y las Tecnologías, Institución Universitaria Politécnico Grancolombiano (Colombia). https://orcid.org/0000-0001-8938-7106

Paola Andrea Zuluaga Ortiz es Magíster en Derecho de los negocios, Candidata a título de Doctorado en Derecho, Abogada, Docente Investigadora del Grupo de Investigación en Derecho Sociedad e Industrias Creativas, Escuela de Derecho y Gobierno, Línea de Investigación Derecho de la Información y las Tecnologías, Institución Universitaria Politécnico Grancolombiano (Colombia). https://orcid. org/0000-0002-3605-5973 